\title{
EXPANSIONS IN SERIES OF NON-ORTHOGONAL FUNCTIONS
}

\author{
R. V. CHURCHILL
}

1. Introduction. Let $\phi_{i}(x)(i=1,2, \cdots)$ be the normalized characteristic functions of the Sturm-Liouville problem

$$
\begin{gathered}
\frac{d}{d x}\left(R \frac{d \phi}{d x}\right)+(\lambda P+Q) \phi=0, \\
A_{0} \phi^{\prime}(0)+B_{0} \phi(0)=0, \quad A_{1} \phi^{\prime}(1)+B_{1} \phi(1)=0,
\end{gathered}
$$

in which the functions $P, Q$, and $R$ are continuous, and $R>0, P>0$, when $0 \leqq x \leqq 1$. The set of functions $\left\{\phi_{i}(x)\right\}$ is closed with respect to the class $L^{2}(0,1)$, in the sense that Parseval's relation,

$$
\int_{0}^{1} P f^{2} d x=\sum_{1}^{\infty}\left[\int_{0}^{1} P f \phi_{i} d x\right]^{2}
$$

is satisfied by every function $f$ of that class. This fact can be deduced readily from a theorem by Kellogg ${ }^{1}$ on the completeness of the set of solutions of the self-adjoint problem of the second order. It can also be obtained from a result found by Dixon. ${ }^{2}$

In terms of two functions $f$ and $G$ of the class $L^{2}(0,1)$, Parseval's relation can be written

$$
\int_{0}^{1} P f G d x=\sum_{1}^{\infty} \int_{0}^{1} P f \phi_{i} d x \int_{0}^{1} P G \phi_{i} d x=\sum_{1}^{\infty} c_{i} \int_{0}^{1} P G \phi_{i} d x,
$$

where $c_{i}$ are the Fourier constants of $f$. If $G=g / P$ when $0<x<t$ and $G \equiv 0$ when $t<x<1$, where the function $g$ belongs to $L^{2}(0,1)$, it follows that

$$
\int_{0}^{t} f g d x=\sum_{1}^{\infty} c_{i} \int_{0}^{t} g \phi_{i} d x, \quad 0 \leqq t \leqq 1 .
$$

Presented to the Society in two parts, the first under the title On an extension of the Sturm-Liouville development on April 14, 1939, and the second under the title Term-wise integration of Sturm-Liouville expansions on April 12, 1940; received by the editors April 25, 1941.

${ }^{1}$ O. D. Kellog, Note on closure of orthogonal sets, this Bulletin, vol. 27 (1920), pp. $165-169$.

2 A. C. Dixon, On the series of Sturm-Liouville, as derived from a pair of fundamental integral equations instead of a differential equation, Philosophical Transactions of the Royal Society of London, (A), vol. 211 (1912), pp. 411-432; pp. 431, 432. 
The convergence of this series is uniform with respect to $t(0 \leqq t \leqq 1)$. For, according to Cauchy's inequality,

$$
\left|\sum_{m}^{n} c_{i} \int_{0}^{t} g \phi_{i} d x\right| \leqq\left[\sum_{m}^{n} c_{i}^{2} \sum_{m}^{n}\left(\int_{0}^{t} g \phi_{i} d x\right)^{2}\right]^{1 / 2}, \quad n>m .
$$

But the Parseval relation is true for the function $G$, so that

$$
\sum_{1}^{\infty}\left(\int_{0}^{t} g \phi_{i} d x\right)^{2}=\int_{0}^{t} \frac{g^{2}}{P} d x \leqq \int_{0}^{1} \frac{g^{2}}{P} d x .
$$

Since the series $\sum_{1}^{\infty} c_{i}^{2}$ converges, the uniform convergence of the series in (1) becomes apparent.

Hence the generalized Fourier series $\sum_{1}^{\infty} c_{i} \phi_{i}(x)$ corresponding to $f$ can be multiplied by $g$ and integrated term by term to give the uniformly convergent representation (1).

It is our purpose to use this integration process to derive an expansion in series of the characteristic functions of the following problem:

$$
\begin{aligned}
y^{\prime \prime}+(\lambda+q) y & =0, \\
a_{0} y(0)+y^{\prime}(0)-b_{0} y^{\prime \prime}(0) & =0, \\
a_{1} y(1)+y^{\prime}(1)+b_{1} y^{\prime \prime}(1) & =0,
\end{aligned}
$$

in which the function $q$ is continuous in the interval $(0,1)$; the $a$ 's and $b$ 's are constants, and $b_{0} \geqq 0, b_{1} \geqq 0$. Problems of this type arise in a variety of boundary problems in partial differential equations. ${ }^{3}$

In view of the differential equation, the boundary conditions can be written

$$
\begin{aligned}
& y^{\prime}(0)+\left[a_{0}+b_{0} q(0)+b_{0} \lambda\right] y(0)=0, \\
& y^{\prime}(1)+\left[a_{1}-b_{1} q(1)-b_{1} \lambda\right] y(1)=0,
\end{aligned}
$$

thus showing how the parameter $\lambda$ is involved in those conditions. ${ }^{4}$ If $\lambda_{i}$ and $\lambda_{j}$ are two distinct characteristic numbers of the system (2), and $y_{i}, y_{j}$ the corresponding characteristic functions, it follows by a familiar procedure that

${ }^{3}$ See, for example, R. E. Langer, $A$ problem in diffusion or in the flow of heat for a solid in contact with a fluid, Tôhoku Mathematical Journal, vol. 35 (1932), pp. 260275 ; S. Timoshenko, Vibration Problems in Engineering, 1928, pp. 209, 217. Expansion theorems for the case $q \equiv 0$ will be found in the first of these references; also see A. Kneser, Integralgleichungen, 1922, pp. 117-121.

${ }^{4}$ Theories have been developed, under hypotheses that are quite different from those with which we are concerned here, applying to general boundary value problems which have the form (2) as a special case. See, for instance, W. T. Reid, American Journal of Mathematics, vol. 54 (1932), pp. 769-790, and J. Tamarkin, Mathematische Zeitschrift, vol. 27 (1927), pp. 1-54. 


$$
\frac{d}{d x}\left(y_{j} y_{i}^{\prime}-y_{i} y_{j}^{\prime}\right)=\left(\lambda_{j}-\lambda_{i}\right) y_{i} y_{j} .
$$

Hence, in view of (3), the characteristic functions of (2) satisfy the modified orthogonality relation

$$
B\left(y_{i}, y_{j}\right)=0,
$$

where

$$
B\left(y_{i}, y_{j}\right)=\int_{0}^{1} y_{i} y_{j} d x+b_{0} y_{i}(0) y_{j}(0)+b_{1} y_{i}(1) y_{j}(1) .
$$

2. An associated Sturm-Liouville problem. Since $b_{0} \geqq 0$ and $b_{1} \geqq 0$, problem (2) has an infinite set of real characteristic numbers $\lambda_{i}$ $\left(i=0,1,2, \cdots ; \lambda_{0}<\lambda_{1}<\lambda_{2}<\cdots\right)$, and the corresponding characteristic function $y_{i}$ has exactly $i$ zeros in the interval $0<x<1$. $^{5}$ According to the conditions (3), none of the functions vanishes at either boundary. We assume henceforth that the sign of $y_{0}(x)$ has been so adjusted that $y_{0}>0$ when $0 \leqq x \leqq 1$.

Now let the functions $Y_{i}$ be defined as follows:

$$
Y_{i}(x)=\frac{d}{d x}\left[\frac{y_{i}(x)}{y_{0}(x)}\right], \quad i=1,2, \cdots .
$$

Then, in view of (4),

$$
\frac{d}{d x}\left(y_{0}^{2} Y_{i}\right)=\left(\lambda_{0}-\lambda_{i}\right) y_{0} y_{i}
$$

and therefore

$$
\frac{d}{d x}\left[\frac{1}{y_{0}^{2}} \frac{d}{d x}\left(y_{0}^{2} Y_{i}\right)\right]+\left(\lambda_{i}-\lambda_{0}\right) Y_{i}=0 .
$$

Replacing $y_{i}^{\prime}$ by $y_{0} Y_{i}+y_{0}^{\prime} y_{i} / y_{0}$ and $y_{i}^{\prime \prime}$ by $y_{0} Y_{i}^{\prime}+2 y_{0}^{\prime} Y_{i}+y_{0}^{\prime \prime} y_{i} / y_{0}$ in the first boundary condition

$$
a_{0} y_{i}(0)+y_{i}^{\prime}(0)-b_{0} y_{i}^{\prime \prime}(0)=0,
$$

we find that the resulting coefficient of $y_{i}(0)$ is $\left[a_{0} y_{0}(0)+y_{0}^{\prime}(0)\right.$ $\left.-b_{0} y_{0}^{\prime \prime}(0)\right] / y_{0}(0)$. This vanishes because $y_{0}$ satisfies the boundary condition. Proceeding in the same way to express the second boundary condition of (2) in terms of $Y_{i}$, and rewriting the differential equation (6), we find that the functions $Y_{i}(x)(i=1,2, \cdots)$ are the

${ }^{5}$ See, for instance, E. L. Ince, Ordinary Differential Equations, 1927, pp. 231-235. 
characteristic functions of the following Sturm-Liouville problem:

$$
\begin{aligned}
\frac{d}{d x}\left(y_{0} \frac{d Y_{i}}{d x}\right)+\left[\lambda_{i} y_{0}^{2}-\lambda_{0} y_{0}^{2}-2\left(y_{0}^{\prime}\right)^{2}+2 y_{0} y_{0}^{\prime \prime}\right] Y_{i} & =0, \\
b_{0} y_{0}(0) Y_{i}^{\prime}(0)-\left[y_{0}(0)-2 b_{0} y_{0}^{\prime}(0)\right] Y_{i}(0) & =0, \\
b_{1} y_{0}(1) Y_{i}^{\prime}(1)+\left[y_{0}(1)+2 b_{1} y_{0}^{\prime}(1)\right] Y_{i}(1) & =0 .
\end{aligned}
$$

The characteristic numbers $\lambda_{1}, \lambda_{2}, \cdots$ of problem (2) are clearly the characteristic numbers of (7) that correspond to the characteristic functions $Y_{1}, Y_{2}, \cdots$. Except possibly for $\lambda_{0}$, every characteristic number of (2) must also belong to this new system; but since all those of (7) are known to be real, all characteristic numbers of (2) must be real.

In order to show that $Y_{i}(x)$ are the only characteristic functions of $(7)$, let $U(x)$ denote a solution corresponding to the value $\mu$ of the parameter written as $\lambda_{i}$ in (7). Let $u(x)$ be defined as follows:

$$
u(x)=y_{0}(x)\left[\int_{0}^{x} U(x) d x+C\right],
$$

where $C$ is a constant yet to be determined. Since $d\left(u / y_{0}\right) / d x=U$, it is easily seen that

$$
\frac{u^{\prime \prime}+(\mu+q) u}{y_{0}}=\frac{1}{y_{0}^{2}} \frac{d}{d x}\left(y_{0}^{2} U\right)+\left(\mu-\lambda_{0}\right) \frac{u}{y_{0}} .
$$

The derivative of the second member of this equation is zero, accord ${ }^{-}$ ing to (6); therefore

$$
u^{\prime \prime}+(\mu+q) u=K y_{0},
$$

where $K$ is some constant; also,

$$
U^{\prime}+2 \frac{y_{0}^{\prime}}{y_{0}} U+\left(\mu-\lambda_{0}\right)\left(\int_{0}^{x} U d x+C\right)=K .
$$

If $\mu \neq \lambda_{0}$, there is one value of $C$ for which $K=0$; for if $x=0$ in (8),

$$
C=-\frac{1}{\mu-\lambda_{0}}\left[U^{\prime}(0)+2 \frac{y_{0}^{\prime}(0)}{y_{0}(0)} U(0)\right] .
$$

By reversing the steps used to obtain the boundary conditions in (7), we find that $u(x)$ satisfies the boundary conditions of the original problem (2). Since $u^{\prime \prime}+(\mu+q) u=0$ when $C$ has the above value, $\mu$ must be one of the characteristic numbers $\lambda_{i}(i=1,2, \cdots)$ of (2). 
Now suppose $\mu=\lambda_{0}$. Let the corresponding function $U$ be denoted by $U_{0}$. According to (8),

$$
U_{0}^{\prime}(x)+2 \frac{y_{0}^{\prime}(x)}{y_{0}(x)} U_{0}(x)=U_{0}^{\prime}(0)+2 \frac{y_{0}^{\prime}(0)}{y_{0}(0)} U_{0}(0) \text {. }
$$

Since $U_{0}$ satisfies the first boundary condition of $(7)$, it follows that

$$
\begin{aligned}
\frac{1}{y_{0}^{2}} \frac{d}{d x}\left(y_{0}^{2} U_{0}\right) & =\frac{U_{0}(0)}{b_{0}} & & \text { if } b_{0} \neq 0, \\
& =U_{0}^{\prime}(0) & & \text { if } b_{0}=0,
\end{aligned}
$$

and, after multiplying through by $y_{0}^{2}$ and integrating, we obtain

$$
\begin{aligned}
y_{0}^{2}(1) U_{0}(1) & =U_{0}(0)\left[y_{0}^{2}(0)+\frac{1}{b_{0}} \int_{0}^{1} y_{0}^{2} d x\right] & & \text { if } b_{0} \neq 0, \\
& =U_{0}^{\prime}(0) \int_{0}^{1} y_{0}^{2} d x & & \text { if } b_{0}=0 .
\end{aligned}
$$

Suppose $b_{0} \neq 0$. In view of the boundary conditions of $(7)$, equation (9) with $x=1$ reduces to $U_{0}(0) / b_{0}=-U_{0}(1) / b_{1}$, if $b_{1} \neq 0$. Therefore $U_{0}(0)$ and $U_{0}(1)$ must be of opposite sign, in contradiction to (10). If $b_{1}=0$ then $U_{0}(1)=0$; but $U_{0}(0) \neq 0$ so that $(10)$ is again contradicted.

When $b_{0}=0$ we find in like manner that $U_{0}^{\prime}(0)=-U_{0}(1) / b_{1}$, if $b_{1} \neq 0$, and this is contrary to (10). If $b_{1}=0$ also, then $U_{0}(1)=0$, and since $U_{0}^{\prime}(0) \neq 0$ the contradiction again appears.

Therefore $\lambda_{0}$ cannot be a characteristic number of the system (7), and the following theorem is established:

THEOREM 1. Let $\lambda_{0}, \lambda_{1}, \lambda_{2}, \cdots$ be the set of characteristic numbers of problem (2), and $y_{0}, y_{1}, y_{2}, \cdots$ the corresponding characteristic functions $\left(y_{0}>0\right)$. Then for the Sturm-Liouville problem ( 7$)$, the complete set of characteristic numbers consists of $\lambda_{1}, \lambda_{2}, \cdots$, and the corresponding characteristic functions $Y_{1}, Y_{2}, \cdots$ are related to those of the first problem in the following way:

$$
y_{i}(x)=y_{0}(x)\left(\int_{0}^{x} Y_{i}(x) d x+C_{i}\right), \quad i=1,2, \cdots,
$$

where

$$
C_{i}=-\frac{1}{\lambda_{i}-\lambda_{0}}\left[Y_{i}^{\prime}(0)+2 \frac{y_{0}^{\prime}(0)}{y_{0}(0)} Y_{i}(0)\right]=\frac{y_{i}(0)}{y_{0}(0)}
$$


3. The expansion theorem. Let $f(x)$ be such that the function $d\left(f / y_{0}\right) / d x$ belongs to the class $L^{2}(0,1)$. The generalized Fourier series corresponding to the latter function, with respect to the functions $Y_{i}$, is

where

$$
\sum_{1}^{\infty} A_{i} Y_{i}(x)
$$

$$
A_{i} \int_{0}^{1} y_{0}^{2} Y_{i}^{2} d x=\int_{0}^{1} y_{0}^{2} \frac{d}{d x}\left(\frac{f}{y_{0}}\right) Y_{i} d x, \quad i=1,2, \cdots .
$$

The formula (1), with $g \equiv 1$, applies to this series. Therefore, in view of the above relation between $Y_{i}$ and $y_{i}$, we can write

$$
\int_{0}^{x} \frac{d}{d x}\left(\frac{f}{y_{0}}\right) d x=\sum_{1}^{\infty} A_{i}\left[\frac{y_{i}(x)}{y_{0}(x)}-\frac{y_{i}(0)}{y_{0}(0)}\right], \quad 0 \leqq x \leqq 1 .
$$

This is an expansion in terms of the non-orthogonal characteristic functions of problem (2). The convergence of the series is uniform.

In order to improve the form of this result, we now assume that the derivative $f^{\prime}(x)$ exists and is bounded when $0 \leqq x \leqq 1$, and that it belongs to the class $L(0,1)$. The derivative of $f / y_{0}$ then belongs to $L^{2}(0,1)$, and (11) can be written

$$
y_{0}(0) f(x)-f(0) y_{0}(x)=\sum_{1}^{\infty} A_{i}\left[y_{0}(0) y_{i}(x)-y_{i}(0) y_{0}(x)\right] .
$$

We can multiply all terms here by $y_{0}(x)$ and integrate over the interval $(0,1)$. The member on the right then becomes, in view of the modified orthogonality property (5),

$$
-\sum_{1}^{\infty} A_{i}\left\{B\left(y_{0}, y_{0}\right) y_{i}(0)+b_{1} y_{0}(1)\left[y_{0}(0) y_{i}(1)-y_{i}(0) y_{0}(1)\right]\right\} \text {. }
$$

Using equation (12) with $x=1$ here, we can write this series as

$$
-B\left(y_{0}, y_{0}\right) \sum_{1}^{\infty} A_{i} y_{i}(0)-b_{1} y_{0}(1)\left[y_{0}(0) f(1)-f(0) y_{0}(1)\right],
$$

so that the result of our operations upon (12) becomes

$$
y_{0}(0) B\left(f, y_{0}\right)-f(0) B\left(y_{0}, y_{0}\right)=-B\left(y_{0}, y_{0}\right) \sum_{1}^{\infty} A_{i} y_{i}(0) .
$$

Hence the series appearing here converges and can be written 


$$
\sum_{1}^{\infty} A_{i} y_{i}(0)=f(0)-A_{0} y_{0}(0)
$$

where

$$
A_{0}=\frac{B\left(f, y_{0}\right)}{B\left(y_{0}, y_{0}\right)} .
$$

The expansion (12) becomes, in view of (13),

$$
f(x)=\sum_{0}^{\infty} A_{i} y_{i}(x), \quad 0 \leqq x \leqq 1 .
$$

The convergence is uniform, since the series in (13) converges and the series in (12) is uniformly convergent.

To express the formula for $A_{i}(i=1,2, \cdots)$ in terms of $y_{i}$, we write

$$
\int_{0}^{1} y_{0}^{2} \frac{d}{d x}\left(\frac{f}{y_{0}}\right) Y_{i} d x=\left[\frac{f}{y_{0}}\left(y_{0} y_{i}^{\prime}-y_{i} y_{0}^{\prime}\right)\right]_{0}^{1}+\left(\lambda_{i}-\lambda_{0}\right) \int_{0}^{1} f y_{i} d x .
$$

Eliminating the boundary values of the derivatives with the aid of (3), we find that the second member here is equal to

$$
\left(\lambda_{i}-\lambda_{0}\right) B\left(f, y_{i}\right) \text {. }
$$

Finally, by replacing $f$ by $y_{i}$, we obtain at once the corresponding expression for $\int_{0}^{1} y_{0}^{2} Y_{i}^{2} d x$. Therefore

$$
A_{i}=\frac{B\left(f, y_{i}\right)}{B\left(y_{i}, y_{i}\right)}, \quad i=0,1,2, \cdots .
$$

We have included $i=0$ here since this formula agrees with the one above for $A_{0}$.

Our result can be stated as follows:

TheOREm 2. Let $f(x)$ be any function whose derivative $f^{\prime}(x)$ exists throughout the interval $(0,1)$ and for which $f^{\prime}(x)$ is bounded and L-integrable in that interval. Then $f(x)$ admits the representation

$$
f(x)=\sum_{0}^{\infty} A_{i} y_{i}(x), \quad 0 \leqq x \leqq 1,
$$

where $y_{i}(x)$ are the characteristic functions of the differential system (2), and the coefficients $A_{i}$ are defined by (14). Moreover the convergence of this series to $f(x)$ is uniform in the interval $0 \leqq x \leqq 1$.

The University of Michigan 